\title{
Prevalência de Hipertensão do Avental Branco na Atenção Primária de Saúde
}

\author{
Prevalence of White Coat Hypertension in Primary Health Care
}

\section{Leila Maria Marchi Alves, Maria Suely Nogueira, Simone de Godoy, Miyeko Hayashida, Evelin Capellari Cárnio}

Escola de Enfermagem de Ribeirão Preto - USP - Ribeirão Preto, SP - Brasil

\section{Resumo}

Objetivo: Avaliar a prevalência de hipertensão do avental branco no município de Dumont, Estado de São Paulo, caracterizando os participantes da pesquisa em relação a fatores demográficos e a alterações tanto fisiológicas como metabólicas.

Métodos: Foram selecionados 109 usuários da Unidade de Saúde municipal, divididos em três grupos (normotensão, hipertensão essencial e hipertensão do avental branco), após medidas de pressão arterial com aparelho oscilométrico e exame de monitorização ambulatorial da pressão arterial. Foram realizadas entrevista, mensuração de dados e coleta de exames laboratoriais para comparação das variáveis encontradas entre os grupos. Para o tratamento estatístico, foram utilizados os testes ANOVA e Tukey. Os resultados foram expressos como médias \pm erros padrão das médias. As diferenças foram consideradas estatisticamente significativas para $\mathrm{p}<0,05$.

Resultados: A prevalência de hipertensão do avental branco foi de 34,1\%, com predominância do sexo feminino, média de idade de 45,3 anos e aumento de índice de massa corporal, relação cintura-quadril, glicemia e creatinina plasmáticas, na comparação com hipertensos e/ou normotensos. Não foi encontrada relação entre hipertensão do avental branco e variáveis demográficas.

Conclusão: As diferenças encontradas entre os grupos e a presença de variações clínicas e bioquímicas possibilitam inferir que a hipertensão do avental branco é uma condição que deve ser analisada de maneira distinta em relação a indivíduos normotensos e hipertensos. (Arq Bras Cardiol 2007; 89(1) : 28-35)

Palavras-chave: Pressão arterial, hipertensão, monitorização ambulatorial da pressão arterial, hipertensão do avental branco.

\section{Summary}

Objective: Assess the prevalence of white coat hypertension in the municipality of Dumont, in São Paulo State, and map study participants' demographics, and physiologic and metabolic changes.

Methods: One hundred and nine (109) users of the Municipal Health Service were selected and then divided into three groups: normotension, essential hypertension, and white coat hypertension after blood pressure was measured through oscilometry and blood pressure monitoring exam at outpatient unit. Variables found between the groups were compared based on interview, data measurements, and laboratory exams. ANOVA and Tukey tests were used for statistical data. Results were expressed as means \pm standard error of means. $p<0.05$ was considered statistically significant.

Results: The prevalence of white coat hypertension was 34.1\%. Females were predominant: mean age 45.3 years, increased body mass index, waist/hip ratio, plasma glucose level and creatinine when compared to hypertensives and/or normotensives. No correlation was found between white coat hypertension and demographic variables.

Conclusion: The differences found between the groups and clinical and biochemical variations lead to the conclusion that white coat hypertension is a condition that should be investigated in normotensive and hypertensive individuals distinctively. (Arq Bras Cardiol 2007; 89(1) : 25-31)

Key words: Blood pressure, hypertension, blood pressure monitoring, ambulatory; white coat hypertension.

Full texts in English - http://www.arquivosonline.com.br

\section{Introdução}

A aferição da pressão arterial constitui-se no mais poderoso indicador de doença hipertensiva, mas é preciso considerar a inerente variabilidade da pressão arterial associada com a

Correspondência: Leila Maria Marchi Alves •

Av. Bandeirantes, 3.900 - 14040-902 - Ribeirão Preto, SP - Brasil E-mail: Imarchi@eerp.usp.br

Artigo recebido em 03/07/06; revisado recebido em 22/11/06; aceito em 17/01/07. medida clínica. Hoje, há evidências inequívocas de que o valor da pressão arterial no ambiente hospitalar comumente difere das cifras encontradas na aferição em outros ambientes e que a discrepância entre esses valores varia de maneira expressiva de um indivíduo para outro. Assim, supõe-se que a pressão arterial ambulatorial retrate de maneira mais fidedigna a cifra pressórica, permitindo identificar o importante subgrupo de pacientes portadores de hipertensão do avental branco ${ }^{1}$.

Hipertensão do avental branco significa elevação persistente da pressão arterial no consultório médico ou na clínica, com 
pressão normal em quaisquer outras circunstâncias quando avaliada pela monitorização ambulatorial da pressão arterial $(\mathrm{MAPA})^{2}$. Esse novo método de aferição da pressão arterial tem sido progressivamente utilizado na prática por sua capacidade de fornecer informações adicionais às usualmente obtidas, a partir dos métodos tradicionais de medida da pressão, além de ser um instrumento que propicia a eliminação de fatores de erro relacionados à aferição e à ampliação das possibilidades diagnósticas e terapêuticas ${ }^{3}$.

Podem ser classificados como hipertensos do avental branco os indivíduos que manifestam hipertensão no consultório, mas mantêm cifras pressóricas normais ao exame de MAPA, conforme proposto por outros autores ${ }^{4-6}$, ressaltando que os critérios utilizados para definir a hipertensão do avental branco são importantes determinantes de sua prevalência e de seu prognóstico ${ }^{7}$. A incidência do fenômeno varia de $20 \%$ a $40 \%$, dependendo desses critérios $^{6-9}$.

Também tem sido analisado se os pacientes com diagnóstico de hipertensão do avental branco podem estar em maior risco de morbidade cardiovascular e se o tratamento medicamentoso é o mais apropriado ${ }^{10}$. Já foi demonstrado que a hipertensão do avental branco pode ser influenciada por vários fatores demográficos ${ }^{11-13}$.

A fisiopatologia da elevação da pressão arterial em hipertensos do avental branco também precisa ser esclarecida ${ }^{14-18}$. Diante desses questionamentos, a proposta deste estudo foi a de avaliar a prevalência de hipertensão do avental branco no município de Dumont, Estado de São Paulo, e caracterizar os participantes da pesquisa em relação a fatores demográficos e a alterações tanto fisiológicas como metabólicas.

\section{Métodos}

Este trabalho foi desenvolvido na cidade de Dumont, município de pequeno porte localizado ao norte do Estado de São Paulo ${ }^{19}$, após ter sido submetido e aprovado pelo Comitê de Ética e Pesquisa da Escola de Enfermagem de Ribeirão Preto da Universidade de São Paulo.

Para a elaboração desta pesquisa, no período de maio de 2004 a janeiro de 2006 foram entrevistados e cadastrados 441 pacientes que compareceram à Unidade de Saúde para consulta e/ou tratamento ambulatorial, sendo em sua maioria hipertensos de longa data em uso de medicamentos, portanto excluídos do estudo.

Grande parte dos indivíduos era proveniente da clínica médica. Não era exigida história prévia de hipertensão, uma vez que o intuito foi o de identificar os diferentes grupos do estudo entre pacientes nunca submetidos a terapia medicamentosa anti-hipertensiva.

Os participantes foram divididos nas seguintes categorias: normotensão - pressão arterial sistólica (PAS) < 135 mmHg e pressão arterial diastólica (PAD) $<85 \mathrm{mmHg}$ no consultório e na média do período de vigília da MAPA; hipertensão essencial - PAS $\geq 135$ mmHg e/ou PAD $\geq 85$ mmHg no consultório e na média do período de vigília da MAPA; hipertensão do avental branco - PAS $\geq 135 \mathrm{mmHg}$ e/ou PAD $\geq 85 \mathrm{mmHg}$ no consultório e PAS $<135 \mathrm{mmHg}$ e PAD $<85 \mathrm{mmHg}$ na média do período de vigília da MAPA.

Inicialmente, o paciente teve seu cadastro preenchido com dados de identificação, história prévia de morbidades, histórico familiar de doença cardíaca, exposição a fatores de risco, antropometria, e medidas de pressão arterial, cintura e quadril. A seguir, foram agendados os procedimentos aos quais os participantes seriam submetidos: exame de MAPA e coleta de sangue para investigação de alterações bioquímicas.

Para a medida da pressão arterial no consultório, pelo método indireto, foram utilizados tensiômetros portáteis Gamma Heine, com braçadeiras adequadas à medida da circunferência braquial do paciente, com alcance de 0 $\mathrm{mmHg}$ a $300 \mathrm{mmHg}$ e precisão de $\pm 3 \mathrm{mmHg}$. Todas as exigências técnicas para a adequada obtenção da pressão arterial, bem como a definição do ponto de corte para a classificação dos sujeitos em hipertensos e normotensos, obedeceram às especificações das IV Diretrizes Brasileiras de Hipertensão Arterial ${ }^{3}$.

O exame de MAPA foi realizado conforme preconizado pelas III Diretrizes para o uso da Monitorização Ambulatorial da Pressão Arterial ${ }^{7}$. Foram utilizados equipamentos Spacelabs/90207 e método oscilométrico, por meio de medida indireta e intermitente, com intervalos de 15 minutos para o período diurno e de 30 minutos para o período noturno. Os exames foram considerados válidos quando tiveram duração mínima de 21 horas, três medidas/hora na vigília e duas medidas/hora no período de sono. Foram excluídos os exames nos quais foram invalidadas $20 \%$ ou mais das medidas automáticas.

Os participantes da pesquisa foram orientados a comparecer à Unidade de Saúde em dia e hora marcados, após jejum de 12 horas, para coleta da amostra de sangue para dosagem sérica de glicemia, colesterol, triglicérides, sódio, potássio, uréia e creatinina. A análise laboratorial dessas amostras ficou a cargo de um laboratório credenciado pela Secretaria Estadual de Saúde - Sistema Único de Saúde (SUS), também utilizado para acompanhamento e tratamento de pacientes cadastrados no Sistema de Cadastramento e Acompanhamento de Hipertensos e Diabéticos (HiperDia), do governo federal ${ }^{20}$.

Para o tratamento estatístico, as variáveis índice de massa corporal (IMC), relação cintura-quadril (RCQ), glicemia, colesterol total, triglicérides, sódio, potássio, uréia e creatinina foram avaliadas utilizando-se análise de variância (ANOVA) para medidas repetidas e teste de Tukey para comparações múltiplas das médias. Os resultados foram expressos como médias \pm erros padrão das médias (EPM). As diferenças foram consideradas estatisticamente significativas para $p<0,05$. O estudo procurou testar se as médias dos grupos diferiam, considerando a variância em todos os grupos.

Este estudo foi financiado pela Fundação de Amparo à Pesquisa do Estado de São Paulo (FAPESP).

\section{Resultados}

Respeitados os devidos critérios de exclusão, foram selecionados para o estudo 109 indivíduos, dos quais 58 $(53,2 \%)$ foram constatados normotensos e 51 (46,8\%) 


\section{Artigo Original}

foram diagnosticados como hipertensos. Destes, 33 (64,7\%) foram classificados como portadores de hipertensão arterial essencial e 18 (35,3\%), como portadores de hipertensão do avental branco.

As médias da PAS e da PAD, verificadas no consultório, estão representadas nas figuras 1 e 2 , respectivamente, e as médias da pressão arterial verificadas ao exame de MAPA, no período de vigília, podem ser observadas nas figuras 3 (PAS) e 4 (PAD).

Do total de 58 pacientes normotensos, 31 (53,4\%) eram do sexo feminino e 27 (46,6\%), do sexo masculino. Em relação aos portadores de hipertensão essencial, 14 (42,4\%) eram do sexo feminino e 19 (57,6\%), do sexo masculino. Dentre os portadores de hipertensão do avental branco, 15

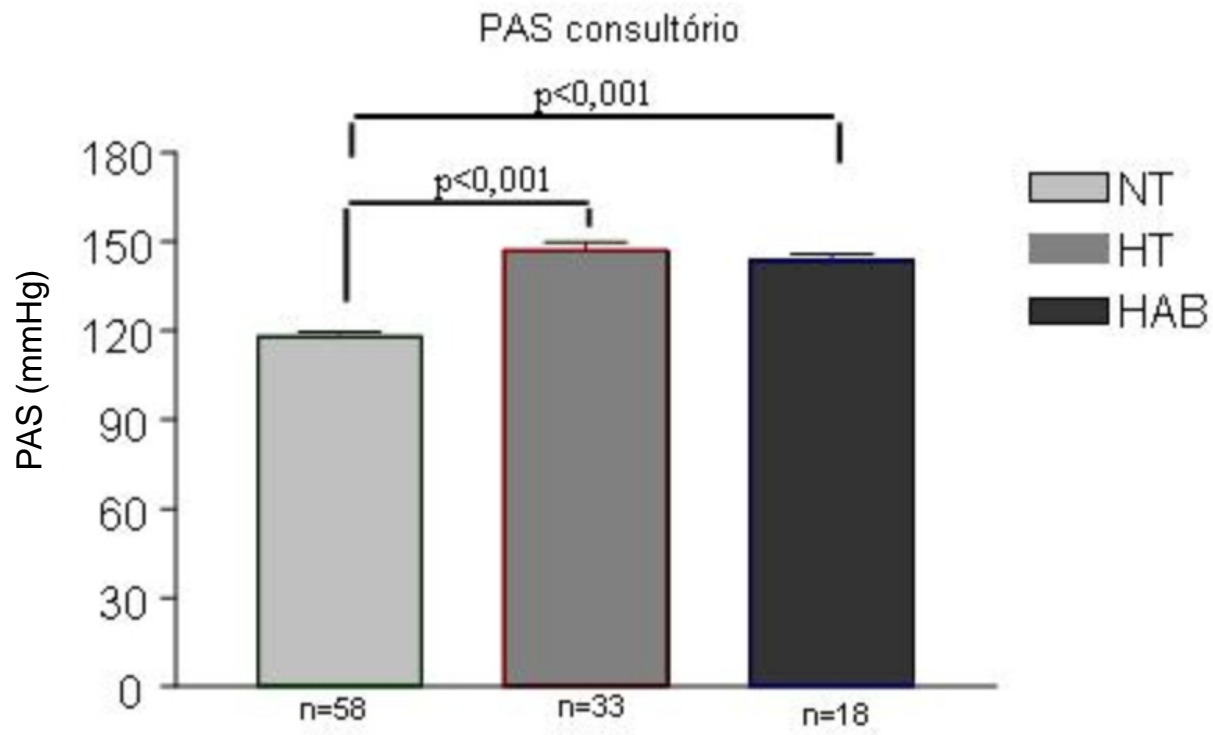

Fig. 1 - Pressão arterial sistólica (PAS) no consultório ( $\mathrm{mmHg}$ ) de indivíduos normotensos (NT), hipertensos essenciais (HT) e hipertensos do avental branco (HAB). Valores expressos como média \pm erros padrão das médias, $n=18$ a 58 em cada grupo. $n$ - número de pacientes.

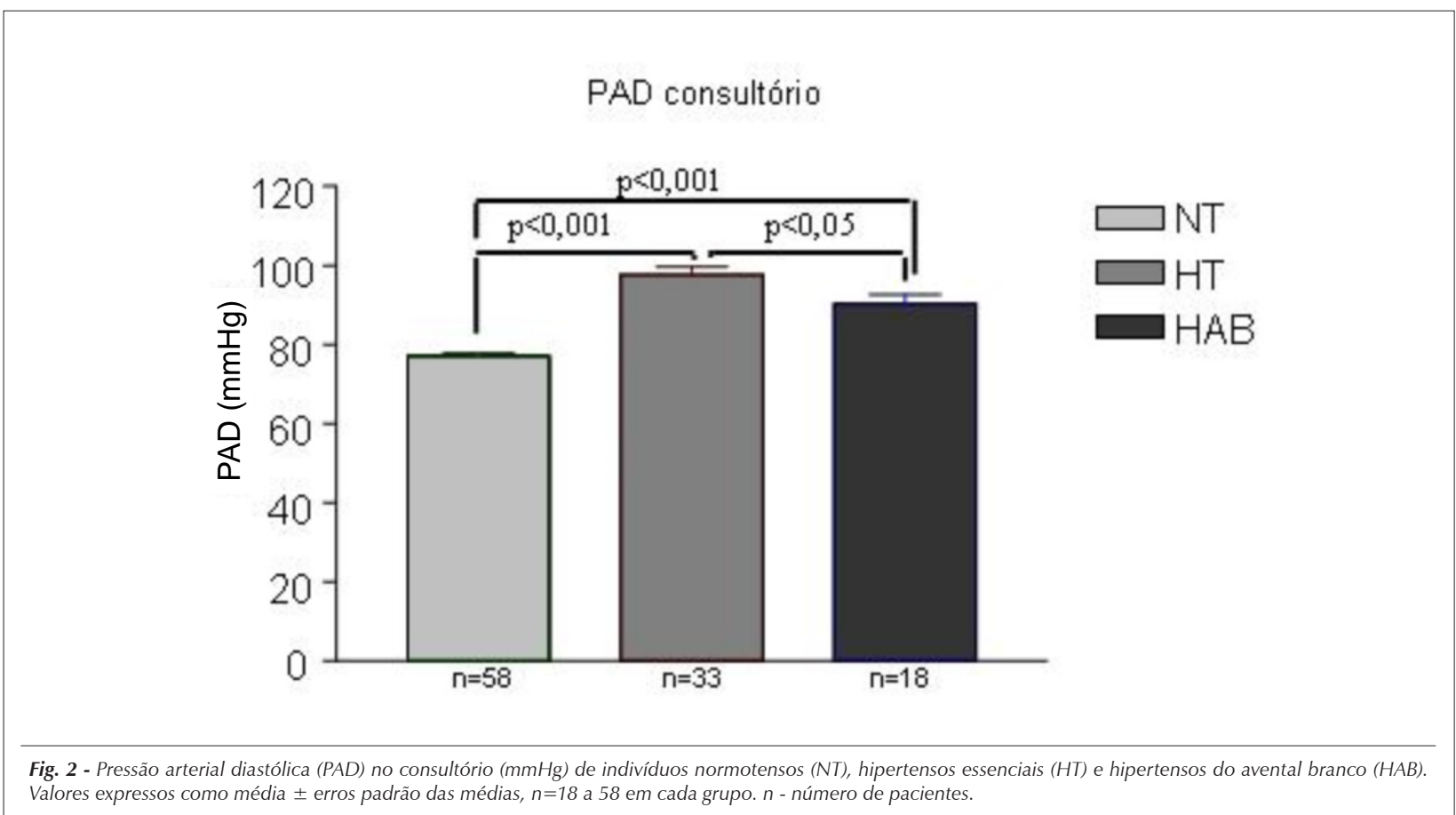




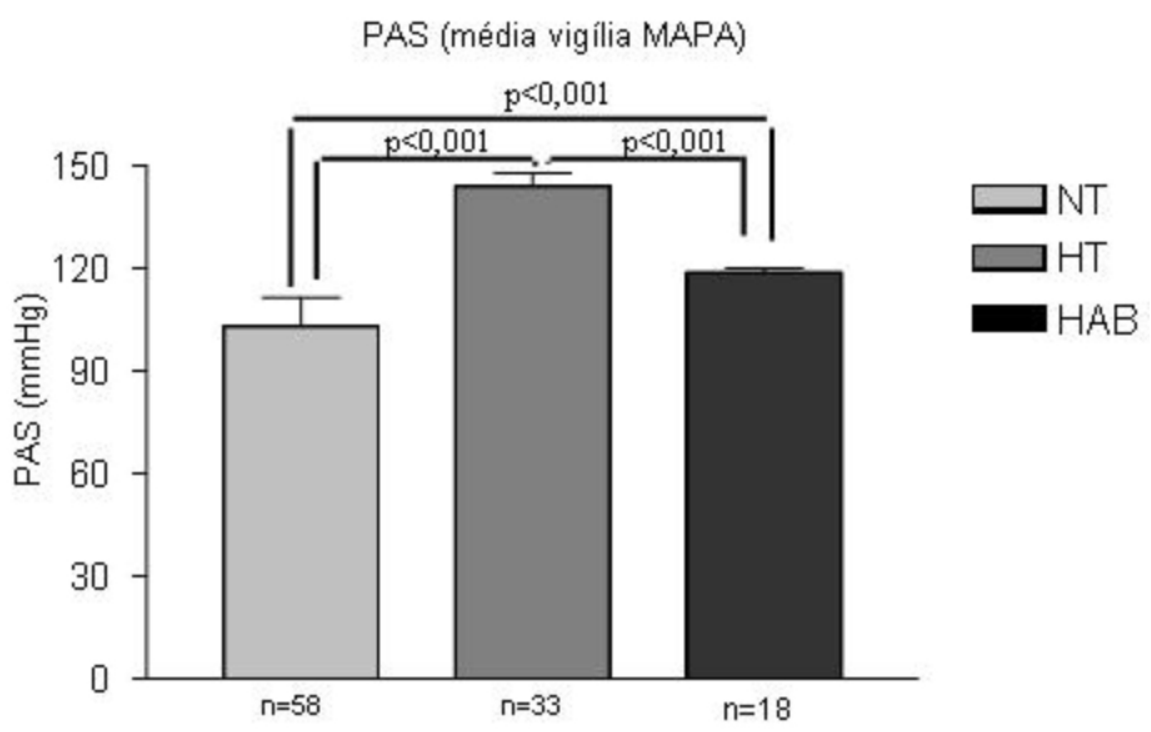

Fig. 3 - Pressão arterial sistólica (PAS) ( $m m H g$ ) na média de vigília da monitorização ambulatorial da pressão arterial (MAPA) de indivíduos normotensos (NT), hipertensos essenciais (HT) e hipertensos do avental branco (HAB). Valores expressos como média \pm erros padrão das médias, $n=18$ a 58 em cada grupo. $n$ - número de pacientes.

PAD (mé dia vigília MAPA)

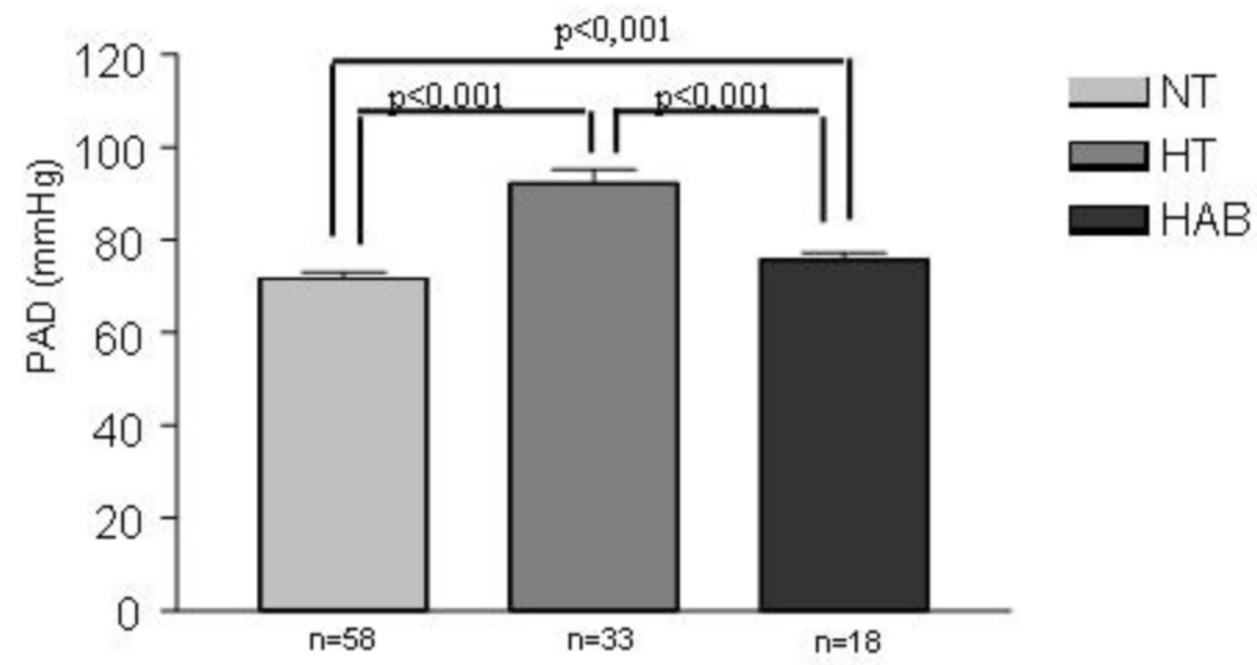

Fig. 4 - Pressão arterial diastólica (PAD) ( $\mathrm{mmHg}$ ) na média de vigília da monitorização ambulatorial da pressão arterial (MAPA) de indivíduos normotensos (NT), hipertensos essenciais (HT) e hipertensos do avental branco (HAB). Valores expressos como média \pm erros padrão das médias, $n=18$ a 58 em cada grupo. $n$ - número de pacientes.

indivíduos (83,3\%) eram do sexo feminino e 3 (16,7\%), do sexo masculino.

Com relação à faixa etária, a maioria dos normotensos (53,4\%) encontrava-se na faixa etária dos 18 aos 29 anos, sendo a média de idade encontrada de 32,2 $\pm 1,6$ anos. Os portadores de hipertensão arterial encontravam-se na faixa de idade entre 50 e 59 anos (33,3\%), com média de idade de 49,26 $\pm 2,7$ anos. Em relação aos hipertensos do avental branco, o maior porcentual $(27,8 \%)$ foi observado na faixa compreendida entre 40 e 49 anos de idade (média de idade, 45,28 $\pm 2,9$ anos).

Achados relacionados ao IMC e à RCQ estão apresentados 
na Tabela 1. Na análise do IMC, se for considerado o padrão internacional indicado pela Associação Brasileira para o Estudo da Obesidade e da Síndrome Metabólica $(\mathrm{ABESO})^{21}$, dentro do peso considerado saudável, 41,2\% eram normotensos, $25,9 \%$ eram hipertensos e $21,4 \%$ eram hipertensos do avental branco. Na faixa de sobrepeso, 35,3\% dos participantes eram normotensos, 29,6\% eram portadores de hipertensão essencial e 35,7\% dos indivíduos eram hipertensos do avental branco. Nos padrões limites, só foi observado baixo peso no grupo normotenso $(7,3 \%)$ e obesidade grau III apenas no grupo avental branco $(7,1 \%)$; os demais pertenciam à categoria de obesidade graus I e II, concentrados no grupo de hipertensos essenciais $(44,4 \%)$ e de hipertensos do avental branco (35,7\%).

Todos os participantes da pesquisa eram procedentes da cidade de Dumont. Observou-se que a maioria dos pacientes era natural do Estado de São Paulo (63,8\% dos normotensos, $66,7 \%$ dos hipertensos e $66,7 \%$ dos hipertensos do avental branco), totalizando $65,1 \%$ da amostra geral. Deve-se ressaltar que $81,7 \%$ dos indivíduos pesquisados eram naturais da Região Sudeste.

Com relação ao nível de escolaridade, a maioria dos sujeitos da pesquisa $(25,7 \%)$ encontrava-se na categoria de alfabetizados funcionais. Também foi significativo o número de sujeitos com ensino médio completo, representando $24,8 \%$ da amostra. De maneira geral, em relação ao nível de escolaridade, no grupo de normotensos foram encontrados os melhores índices (62,3\% dos participantes concluíram o ensino fundamental e $34,3 \%$ completaram o ensino médio) e no grupo de hipertensos foram observados os piores indicadores, em que $78,6 \%$ dos sujeitos completaram no máximo o ensino fundamental e 36,3\% eram apenas alfabetizados.

Na consideração do estado civil, verificou-se que a maioria dos indivíduos da pesquisa era casada, tanto na amostra global $(64,2 \%)$ como em cada uma das categorias. Contudo, dentre os hipertensos, os indivíduos casados representavam quase a totalidade dos pesquisados (93,9\%).

Histórico familiar de hipertensão esteve presente em
$69 \%$ dos normotensos, em 78,8\% dos hipertensos e em $72,2 \%$ dos portadores de hipertensão do avental branco. Os demais negaram ou informaram desconhecer antecedentes familiares.

Na Tabela 1 também estão apresentados os valores da dosagem sérica em jejum de glicemia, colesterol total, triglicérides, sódio, potássio, uréia e creatinina.

\section{Discussão}

Foi referido, inicialmente, que, dependendo dos critérios do estudo, a prevalência de hipertensão do avental branco pode variar 22,23 . Os resultados deste estudo estão dentro dos limites estabelecidos pela literatura, uma vez que 35,3\% dos hipertensos foram diagnosticados como avental branco.

Os índices pressóricos dos indivíduos estudados definiram consistentemente os grupos de normotensos e hipertensos. Foi observada diferença significativa nos valores de pressão arterial, tanto sistólica como diastólica, entre os indivíduos pertencentes aos distintos grupos, na medida de consultório e na média do período de vigília da MAPA. É interessante observar que, em relação à PAS mensurada no consultório, os grupos de hipertensos guardaram semelhança entre si. Se for considerado que o aumento da pressão arterial é importante preditor de risco para doenças cardiovasculares ${ }^{24}$, esse achado sugere que os hipertensos do avental branco estão em maior risco para o evento quando comparados a indivíduos com pressão arterial controlada ou podem apresentar exposição tão intensa quanto o portador de hipertensão primária.

Em relação ao sexo, somente no subgrupo hipertensão do avental branco houve predominância de participantes do sexo feminino, corroborando os resultados de muitos pesquisadores $^{11,25}$, que apontam para maior freqüência do fenômeno entre mulheres.

No que se refere à distribuição dos sujeitos de acordo com a faixa etária, a prevalência de indivíduos jovens no grupo de normotensos pode ser justificada pela maior incidência de eventos patológicos diretamente associados à progressão

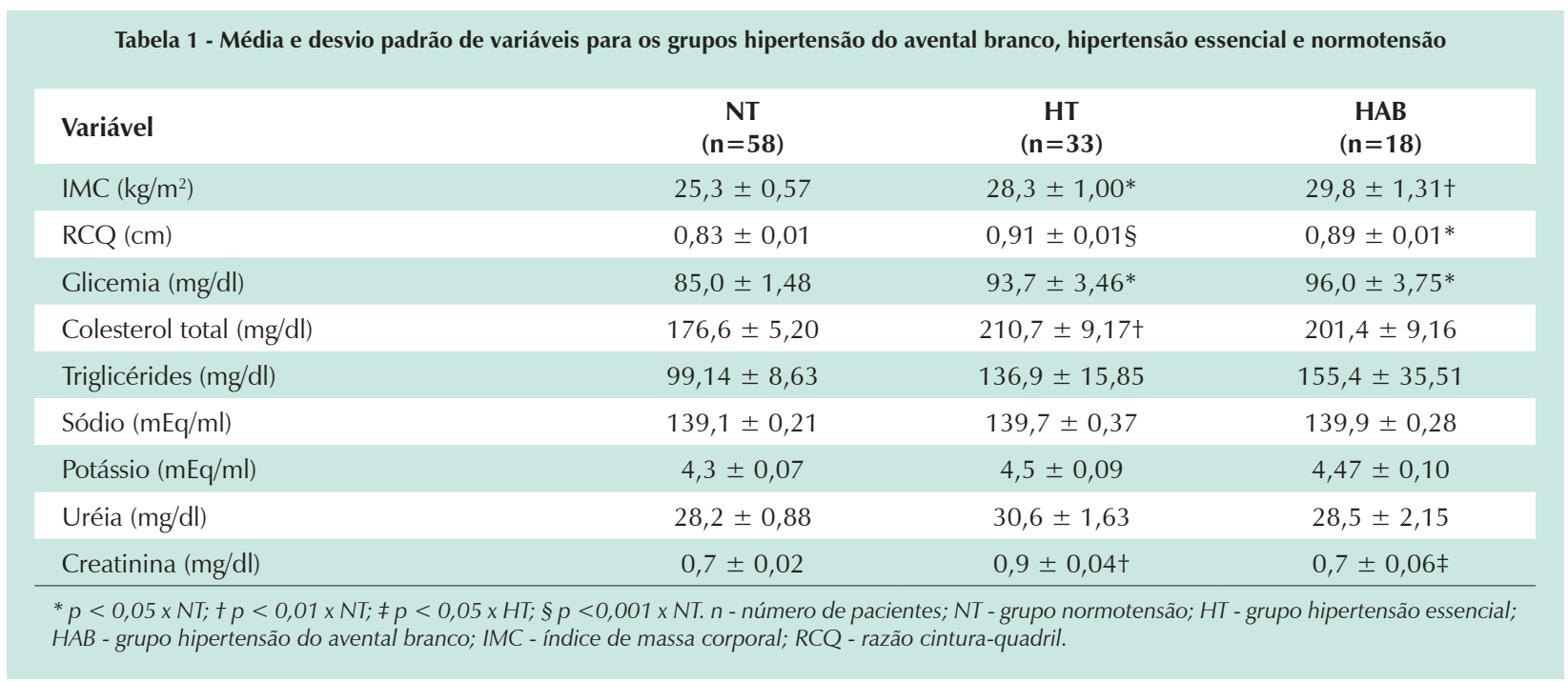


da idade, o que leva ao aumento da faixa etária nos grupos relativos à hipertensão. Cientes da vulnerabilidade de estudos dessa natureza em relação a possíveis vieses, especialmente de seleção, foi feita uma tentativa de pareamento para tornar os casos e controles comparáveis com respeito a fatores constitucionais importantes. No entanto, ao se considerar determinados constituintes, como a idade dos participantes, partindo de uma amostra aleatória, é bastante evidente que as menores cifras pressóricas estejam diretamente associadas às menores faixas etárias. Partindo do princípio de que para amenizar esse viés seria preciso arbitrar sobre o plano amostral de maneira intencional, optou-se por manter a distribuição dos participantes conforme a apresentação original.

Dessa forma, os dados estão de acordo com os índices encontrados na população em geral, em que se constata tendência de aumento da pressão arterial com a idade e elevada incidência de hipertensão arterial entre os idosos ${ }^{3}$.

A maioria dos sujeitos era procedente da Região Sudeste, embora as cidades de origem dos participantes da pesquisa fossem diversificadas. Isso se explica, de maneira geral, pelo fato de os moradores da localidade serem nascidos e registrados em cidades circunvizinhas, em decorrência da maneira como está articulado o sistema de referenciamento e encaminhamento de pacientes para atendimento hospitalar. A maior parte da amostra era formada por sujeitos que trabalhavam na agroindústria canavieira ou do amendoim (rurícolas, selecionadores de grãos, fabricantes de doces).

Embora no grupo dos portadores de hipertensão essencial tenham sido observados os menores índices de escolaridade, as diferenças encontradas parecem estar relacionadas com a faixa etária e não com a doença. Ou seja, quanto maior a faixa etária, como a encontrada no grupo de hipertensos, menor o índice de escolaridade. A associação inversa também é verdadeira. Esses resultados corroboram os dados do Instituto Brasileiro de Geografia e Estatística (IBGE) ${ }^{19}$, apontando que no Brasil, na faixa etária dos 45 aos 59 anos, o índice de analfabetismo é significativamente maior quando comparado ao dos jovens. Apesar dessa observação, Martinez e cols. ${ }^{11}$ encontraram, à semelhança do achado deste estudo, que hipertensos do avental branco têm menor nível educacional em comparação com não-hipertensos.

Considerando o estado civil, a quase totalidade dos hipertensos e a maior parte dos portadores de hipertensão do avental branco são constituídas por indivíduos casados ou que apresentam junção marital estável. Alguns estudos ${ }^{26,27}$ apontam que estressores psicossociais, como a união conjugal e os fatores vinculados a essa condição, estão diretamente associados ao aumento da pressão arterial.

Foi encontrada alta prevalência de antecedentes familiares para doenças cardiovasculares nos três grupos estudados, ou seja, neste estudo não houve associação estatisticamente significante entre a hipertensão do avental branco e os antecedentes familiares investigados. A literatura aponta que história familiar de hipertensão arterial apresenta associação com a hipertensão do avental branco ${ }^{14,28}$, porém ainda não se sabe qual o papel da história familiar na gênese do aumento pressórico associado ao fenômeno ${ }^{6}$.

Tabagismo pregresso aparece de maneira expressiva em ambos os grupos de hipertensos e tem baixa incidência entre os normotensos. Já o hábito de ingestão de bebidas alcoólicas esteve presente apenas na história dos portadores de hipertensão essencial. Embora tenha sido encontrada associação positiva entre esses fatores e os eventos descritos, sabe-se que os riscos diminuem de maneira proporcional ao tempo de abandono dos vícios ${ }^{29}$ e todos os participantes referidos fumantes e alcoólatras prévios afirmaram ser abstêmios de longa data.

Na análise do IMC, a exemplo do demonstrado por outras investigações ${ }^{12,30}$, os indivíduos de ambos os grupos de hipertensos eram mais obesos que os normotensos, não havendo diferença estatisticamente significativa entre hipertensos do avental branco e hipertensos essenciais.

Ao se avaliar a RCQ para identificação de risco de doenças metabólicas e cardiovasculares ${ }^{31}$, foi encontrado que participantes hipertensos apresentaram maiores índices dessa medida antropométrica em relação aos sujeitos normotensos, estando os hipertensos do avental branco expostos ao risco com a mesma intensidade que o grupo portador de hipertensão essencial.

Já foi referido que fatores de risco metabólico relacionados à hipertensão contribuem para o desenvolvimento de lesões de órgãos-alvo e doenças ateroscleróticas. A hipertensão está associada a metabolismo anormal de glicose e dislipidemia. De maneira semelhante, muitos estudos indicam distúrbios metabólicos na hipertensão do avental branco, mas os resultados são inconsistentes ${ }^{29,32}$. Esta pesquisa demonstrou que a glicemia plasmática de indivíduos hipertensos de ambos os grupos é maior comparativamente à dos normotensos, ou seja, portadores de hipertensão do avental branco são similares aos portadores de hipertensão essencial na avaliação desse parâmetro, tal qual observado no estudo de Björklund e cols. ${ }^{33}$. A mesma discrepância em relação aos normotensos também foi encontrada na avaliação da creatinina plasmática.

Assim como demonstrado por Sandvik e Steine ${ }^{34}$, a dosagem sérica de colesterol total indicou maior nível lipídico em pacientes com hipertensão essencial quando comparados a normotensos, mas a diferença não foi significativa para hipertensos do avental branco. Apesar disso, a média de valores para ambos os grupos de hipertensos está acima do nível ideal, considerando-se a classificação de risco cardiovascular e as metas de controle do perfil lipídico ditadas pelas Diretrizes Brasileiras de Hipertensão Arterial ${ }^{3}$. Esse dado sugere que, embora graficamente comparáveis aos sujeitos sem alteração da pressão arterial, ao se avaliar os índices lipêmicos os hipertensos do avental branco também estão expostos aos riscos deletérios ocasionados pela hipercolesterolemia.

Não foi encontrada diferença estatisticamente significativa entre os três grupos em relação aos níveis séricos de triglicérides, sódio, potássio e uréia. Interessante notar que, considerando-se a média dos valores para a dosagem de triglicérides isoladamente, o grupo portador de hipertensão do avental branco apresentou não somente médias mais elevadas que os demais, mas surpreendentemente foi o único grupo com resultados acima dos parâmetros de normalidade encontrados na literatura.

Os resultados deste estudo apontam que os riscos podem 
tanto estar próximos aos apresentados por portadores de hipertensão essencial como serem comparáveis aos sujeitos normotensos, fortalecendo o conceito de que os hipertensos do avental branco se encontram em situação de risco intermediária entre hipertensos essenciais e indivíduos sem alteração do quadro pressórico. Mas a literatura apresenta resultados controversos na análise dessas variáveis ${ }^{6,12,14,23,35-38}$.

Acredita-se que as diferenças encontradas entre os grupos em relação a variações clínicas e bioquímicas indicam que a hipertensão do avental branco é uma condição que deve ser analisada de forma diferenciada em relação à normotensão e à hipertensão essencial. Os resultados deste estudo corroboram os de estudos prévios ${ }^{22,39}$, segundo os quais a presença dessas anormalidades deve possibilitar inferir que há aumento de lesões de órgãos-alvo associado à hipertensão do avental branco.

Embora não tenha sido encontrada relação entre hipertensão do avental branco e as variáveis demográficas estudadas, sabe-se que a casuística é pequena para conclusões mais sólidas.

Há carência de estudos dessa natureza em nosso meio, que poderiam ajudar a dimensionar a prevalência e os riscos associados à ocorrência do fenômeno do avental branco. Ponderando todas as particularidades discutidas até

\section{Referências}

1. Pickering TG. Can ambulatory blood pressure monitoring improve the diagnosis of mild hypertension? Curr Sci. 1990; 8 (Suppl 6): S43-S47.

2. Pickering TG. White coat hypertension. Curr Opin Nephrol Hypertens. 1996; 5 (2): 192-8.

3. IV Diretrizes Brasileiras de Hipertensão Arterial. Arq Bras Cardiol. 2004; 82 (supl. 4): 1- 40.

4. Pierdomenico SD, Cipollone F, Lapenna D, Bucci A, Cuccurullo F, Mezzetti A. Endothelial function in sustained and white coat hypertension. Am J Hypertens. 2002; 15 (11): 946-52.

5. Gus M. Que valores devem ser adotados como limites de normalidade na Monitorização Ambulatorial da Pressão Arterial e na Monitorização Residencial da Pressão Arterial? Arq Bras Cardiol. 2005; 85 (3): 212-4.

6. Segre CA, Ueno RK, Warde KRJ, Accorsi TAD, Miname MH, Chi CK, et al. Efeito hipertensão e normotensão do avental branco na Liga de Hipertensão do Hospital das Clínicas, FMUSP: prevalência, características clínicas e demográficas. Arq Bras Cardiol. 2003; 80 (2): 122-6.

7. III Diretrizes Brasileiras para o uso da Monitorização Ambulatorial da Pressão Arterial. Arq Bras Cardiol. 2001; 77 (4): 390-3.

8. O'Brien E, Murphy J, Tyndall A, Atkins N, Mee F, McCarthy G, et al. Twentyfour-hour ambulatory blood pressure in men and women aged 17 to 80 years: the Allied Irish Bank Study. J Hypertens. 1991; 9 (4): 355-60.

9. Hoegholm A, Kristensen KS, Madsen NH, Syendsen TL. White coat hypertension diagnosed by 24-h ambulatory monitoring: examination of 159 newly diagnosed hypertensive patients. Am J Hypertens. 1992; 5 (2): 64-70.

10. Pickering TG. White coat hypertension - should it be treated or not? Cleve Clin J Med. 2002; 69 (8): 584-5.

11. Martinez MA, Garcia-Puig J, Martin JC, Guallar-Castillon P, Aguirre de Carcer A, Torre A, et al. Frequency and determinants of white coat hypertension in mild to moderate hypertension: a primary care-based study. Monitorizacion Ambulatoria de la Presion Arterial (MAPA)-Area 5 Working Group. Am J Hypertens. 1999; 12 (3): 251-9. aqui, acredita-se que precisam ser estabelecidas estratégias relacionadas à utilização de medidas terapêuticas para o controle da pressão arterial nesse paciente. É preciso que sejam dimensionadas a extensão e a probabilidade de associação da hipertensão do avental branco a fatores de risco e co-morbidades, além de ser inegável a necessidade de estudos adicionais para reavaliar e possivelmente definir o melhor encaminhamento frente à manifestação desse fenômeno.

\section{Potencial Conflito de Interesses}

Declaro não haver conflitos de interesses pertinentes.

\section{Vinculação Acadêmica à Pós-Graduação}

Este estudo é parte de tese de doutorado apresentada à Faculdade de Enfermagem de Ribeirão Preto da Universidade de São Paulo.

\section{Fontes de Financiamento}

Esse estudo foi financiado através de bolsa da Fundação de Amparo à Pesquisa do Estado de São Paulo - FAPESP.
12. Julius S, Jamerson K, Gudbrandsson T, Schork N. White coat hypertension: a follow-up. Clin Exp Hypertens A. 1992; 14 (1-2): 45-53.

13. Lemne C, Lindvall K, Georgiades A, Fredrikson M, de Faire U. Structural cardiac changes in relation to $24 \mathrm{~h}$ ambulatory blood pressure levels in borderline hypertension. J Intern Med. 1995; 238 (1): 49-57.

14. Hoegholm A, Bang LE, Kristensen KS, Nielsen JW, Holm J. Microalbuminuria in 411 untreated individuals with established hypertension, white coat hypertension, and normotension. Hypertension. 1994; 24 (1): 101-5.

15. Cerasola G, Cottone S, Nardi E, D'Ignoto G, Volpe V, Mule G, et al. White coat hypertension and cardiovascular risk. J Cardiovasc Risk. 1995; 2 (6): 545-9.

16. Chang NC, Lai ZY, Chan P, Wang TC. Left ventricular filling profiles in young white-coat hypertensive patients without hypertrophy. Hypertension. 1997; 30 (3Pt2): 746-52.

17. Floras JS, Jones JV, Hassan MO, Osikowska B, Sever PS, Sleight P. Cuff and ambulatory blood pressure in subjects with essential hypertension. Lancet. 1981; 2 (8238): 107-9.

18. Smith PA, Graham LN, Mackintosh AF, Stoker JB, Mary DA. Sympathetic neural mechanisms in white coat hypertension. J Am Coll Cardiol. 2002; 40 (1): 126-32.

19. Ministério do Planejamento, Orçamento e Gestão [homepage na internet]. Instituto Brasileiro de Geografia e Estatística. Banco de Dados [citado 2005 dez 14]. Disponível em: < http://www.ibge.gov.br>

20. Ministério da Saúde [homepage na Internet]. Secretaria Executiva. Datasus. Informações de Saúde. Indicadores Municipais de Saúde. [citado 2006 jan 05]. Disponível em: http://tabnet.datasus.gov.br/tabdata/cadernos/ cadernosmap.htm.

21. Associação Brasileira para o Estudo da Obesidade e da Síndrome Metabólica [homepage na internet]. Cálculo de IMC [citado 2005 set 17]. Disponível em: $<$ http://www.abeso.org.br>.

22. Tsai PS. White coat hypertension: understanding the concept and examining the significance. J Clin Nurs. 2002; 11 (6): 715-22. 
23. Celis H, Fagard RH. White-coat hypertension: a clinical review. Eur J Intern Med. 2004; 15 (6): 348-57.

24. Papademetriou V. Comparative prognostic value of systolic, diastolic, and pulse pressure. Am J Cardiol. 2003; 91 (4): 433-5.

25. Myers MG, Reeves RA. White coat effect in treated hypertensive patients: sex differences. J Hum Hypertens. 1995; 9 (9): 729-33.

26. Baker B, Helmers K, O’Kelly B, Sakinofsky I, Abelsohn A, Tobe S. Marital cohesion and ambulatory blood pressure in early hypertension. Am J Hypertens. 1999; 12 (2 Pt 1): 227-30.

27. Tobe SW, Kiss A, Szalai JP, Perkins N, Tsigoulis M, Baker B. Impact of job and marital strain on ambulatory blood pressure results from the double exposure study. Am J Hypertens. 2005; 18 (8): 1046-51.

28. Julius S, Mejia A, Jones K, Krause L, Schork N, van de Ven C, et al. White coat versus sustained borderline hypertension in Tecumseh, Michigan. Hypertension. 1990; 16 (6): 617-23.

29. Eliasson B, Hjalmarson A, Kruse E, Landfeldt B, Westin A. Effect of smoking reduction and cessation on cardiovascular risk factors. Nicotine Tob Res. 2001; 3 (3): 249-55.

30. Kotsis V, Stabouli S, Bouldin M, Low A, Toumanidis S, Zakopoulos N. Impact of obesity on 24-hour ambulatory blood pressure and hypertension. Hypertension. 2005; 45 (4): 602-7.

31. Yusuf S, Hawken S, Ounpuu S, Bautista L, Franzosi MG, Commerford P, et al. Obesity and the risk of myocardial infarction in 27,000 participants from 52 countries: a case-control study. Lancet. 2005; 366 (9497): 1640-9.
32. Marchesi E, Perani G, Falaschi F, Negro C, Catalano O, Ravetta V, et al. Metabolic risk factors in white coat hypertensives. J Hum Hypertens. 1994; 8 (7): 475-9.

33. Björklund K, Lind L, Vessby B, Andren B, Lithell H. Different metabolic predictors of white-coat and sustained hypertension over a 20-year follow-up period: a population-based study of elderly men. Circulation. 2002; 106 (1): 63-8.

34. Sandvik E, Steine S. White coat hypertension in a general practice: prevalence, cardiovascular risk factors and clinical implications. Scand Health Care. 1998; 16 (4): 222-6.

35. Gosse P, Promax H, Durandet P, Clementy J. White coat hypertension: no harm for the heart. Hypertension. 1993; 22 (5): 766-70.

36. Weber MA, Neutel JM, Smith DH, Graettinger WF. Diagnosis of mild hypertension by ambulatory blood pressure monitoring. Circulation. 1994; 90 (5): 2291-8.

37. Cavallini MC, Roman MJ, Pickering TG, Schwartz JE, Pini R, Devereux RB. Is white coat hypertension associated with arterial disease or left ventricular hypertrophy? Hypertension. 1995; 26 (3): 413-9.

38. Rizzo V, Cicconetti P, Bianchi A, Lorido A, Morelli S, Vetta F, et al. White-coat hypertension and cardiac organ damage in elderly subjects. J Hum Hypertens. 1996; 10 (5): 293-8

39. Kuwajima I, Suzuki Y, Fujisawa A, Kuramoto K. Is white coat hypertension innocent? Structure and function of the heart in the elderly. Hypertension. 1993; 22 (6): 826-31. 